\title{
Thermodynamic and physical properties of Zr3Fe and ZrFe2 intermetallic compounds
}

B. O. Mukhamedov, I Saenko, A. V Ponomareva, M. J. Kriegel, A. Chugreev, A. Udovsky, O. Fabrichnaya and Igor Abrikosov

The self-archived postprint version of this journal article is available at Linköping University Institutional Repository (DiVA):

http://urn.kb.se/resolve?urn=urn:nbn:se:liu:diva-157517

N.B.: When citing this work, cite the original publication.

Mukhamedov, B. O., Saenko, I, Ponomareva, A. V, Kriegel, M. J., Chugreev, A., Udovsky, A.,

Fabrichnaya, O., Abrikosov, I., (2019), Thermodynamic and physical properties of $\mathrm{Zr} 3 \mathrm{Fe}$ and $\mathrm{ZrFe} 2$ intermetallic compounds, Intermetallics (Barking), 109, 189-196.

https://doi.org/10.1016/j.intermet.2019.01.018

Original publication available at:

https://doi.org/10.1016/j.intermet.2019.01.018

Copyright: Elsevier

http://www.elsevier.com/

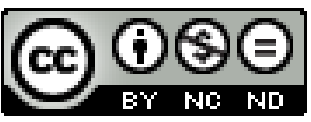




\section{Thermodynamic and physical properties of $\mathrm{Zr}_{3} \mathrm{Fe}$ and $\mathrm{ZrFe}_{2}$ intermetallic compounds}

B.O. Mukhamedov ${ }^{1, *}$, I. Saenko ${ }^{2,3}$, A.V. Ponomareva1, M.J. Kriegel ${ }^{2}$, A. Chugreev ${ }^{4}$, A. Udovsky $^{3,5}$, O. Fabrichnaya ${ }^{2}$, I.A. Abrikosov 6

\footnotetext{
${ }^{1}$ National University of Science and Technology 'MISIS', Moscow, 119049, Russia

${ }^{2}$ Freiberg University of Mining and Technology, Freiberg, Sachsen, 09599, Germany

${ }^{3}$ Baikov Institute of Metallurgy and Material Science of the Russian Academy of Sciences, Moscow, 119991, Russia

${ }^{4}$ IFUM - Institute of Forming Technology and Machines, Leibniz Universitaet Hannover, An der Universitaet 2 , Garbsen 30823, Germany

${ }^{5}$ National Research Nuclear University MEPhI, Moscow, 115409, Russia

${ }^{6}$ Linköping University, Linköping, 581 83, Sweden

*Corresponding author:

B.O. Mukhamedov, Materials Modeling and Development Laboratory, National University of Science and Technology 'MISIS', Moscow, Russia, 119049

E-mail address: b.o.mukhamedov@gmail.com
}

Experimental differential scanning calorimetry measurements and ab-initio simulations were carried out

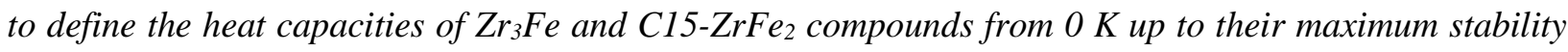
temperatures. Experimental measurements of heat capacity of each compound were performed for the first time in wide range of temperatures. Density functional theory and quasi-harmonic approximation (QHA) were employed to calculate the free energy of the studied systems as a function of volume and temperature. A good agreement was observed between theoretical and experimental heat capacities within validity range of the QHA. This makes it possible to combine theoretical and experimental data to determine the standard entropies of intermetallic compounds.

\section{Introduction}

Zr-based alloys, so-called zircaloys, containing small amount of $\mathrm{Fe}$ and other alloying elements are widely used in nuclear industry due to their low absorption cross-section of thermal neutrons, high corrosion resistance and hardness [1-3]. Moreover, Zr-Fe intermetallic compounds in zircaloys are known to improve specific mechanical properties. Because of this, the $\mathrm{Zr}-\mathrm{Fe}$ system plays an important role in modern steel design. For example, in the manufacturing of composite materials consisting of TRIP (transformation-induced plasticity) matrix and reinforcing $\mathrm{ZrO}_{2}$ based ceramic, the phase relations in the $\mathrm{Zr}-\mathrm{Fe}-\mathrm{O}$ system are essential for the stability of ceramic particles [4]. In fact, both TRIP matrix and ceramic particles may undergo martensitic transformation during compression, which thereby improves the mechanical properties of composite material, such as strength and energy absorption during compression [5].

Therefore, Zr-Fe system is becoming the subject of intense research, and the detailed knowledge on its thermodynamic properties and phase relations is essential for the modern industry of composite and structural steels. A systematic experimental study on binary Zr-Fe system was performed in Ref. [2] for the whole range of compositions. The authors reported that there were four stable intermetallic compounds in the phase diagram: two polymorphic modifications of $\mathrm{ZrFe}_{2}$ phase, $\mathrm{Zr}_{3} \mathrm{Fe}$ and high-temperature $\mathrm{Zr}_{2} \mathrm{Fe}$. Cubic $\mathrm{ZrFe}_{2} \mathrm{C} 15$-Laves phase, which is stable 
up to $1503 \mathrm{~K}$, transforms into hexagonal C36 polymorph at $1563 \mathrm{~K}$ [2]. Similar result was observed in Ref. [6], where the authors found the hexagonal Laves phase in $\mathrm{Zr}_{22.5} \mathrm{Fe}_{77.5}$ alloy after annealing at $1523 \mathrm{~K}$. Intermetallic $\mathrm{Zr}_{3} \mathrm{Fe}$ phase was found to be orthorhombic with $\mathrm{Re}_{3} \mathrm{~B}$ type symmetry [2]. The $\mathrm{Zr}_{3} \mathrm{Fe}$ phase is formed from peritectoid reaction $\mathrm{Zr}_{2} \mathrm{Fe}+\beta-\mathrm{Zr} \rightleftarrows \mathrm{Zr}_{3} \mathrm{Fe}$; and the reported temperature of this reaction varied in several measurements: values of $1124 \mathrm{~K}$ [2], $1158 \mathrm{~K}$ [7], and $1213 \mathrm{~K}$ [8] were reported. Another intermetallic compound $\mathrm{Zr}_{2} \mathrm{Fe}$ is a constituent of above mentioned peritectoid reaction; it is a high-temperature phase with $\mathrm{Ti}_{2} \mathrm{Ni}$ type crystalline structure.

According to the several thermodynamic assessments $[9,10]$, there is limited available information on thermodynamic properties of intermetallic compounds in binary $\mathrm{Zr}$-Fe system. In Ref. [11] the enthalpy of formation $\Delta H_{f}$ of $\mathrm{ZrFe}_{2}$ at $298 \mathrm{~K}$ was determined using direct reaction calorimetry. Enthalpy of formation of $\mathrm{ZrFe}_{2}$ was also derived from indirect measurements by solution calorimetry in Refs. [12], [13] and levitation alloying calorimetry in Ref. [14]. The values of $\Delta H_{f}$ from Refs. [11] and [14] are quite consistent with each other, while the values of $\Delta H_{f}$ in Refs. [12] and [13] are more positive. Several ab-initio calculations predicted fairly consistent results on formation enthalpy of $\mathrm{Zr}-\mathrm{Fe}$ intermetallics: for $\mathrm{C} 15-\mathrm{ZrFe}_{2}$ phase the Density Functional Theory (DFT) calculations gave $-0.280 \mathrm{eV} /$ atom $(-27.02 \mathrm{~kJ} / \mathrm{mol})$ [15] and -0.290 eV/atom $(-27.98 \mathrm{~kJ} / \mathrm{mol})$ [16]. These results are in a good agreement with experimental data from Refs. [11] and [14]. For $\mathrm{Zr}_{3} \mathrm{Fe}$ phase the theoretical enthalpy of formation $\Delta H_{f}$ was found to be equal to $-0.140 \mathrm{eV} /$ atom $(-13.51 \mathrm{~kJ} / \mathrm{mol})$ [15] and $-0.150 \mathrm{eV} / \mathrm{atom}(-14.47 \mathrm{~kJ} / \mathrm{mol})$ [16], however, there were no available experimental results. Regarding the heat capacity, in Ref. [17] the experimental measurements were performed for $\mathrm{C} 15-\mathrm{ZrFe}_{2}$ phase in the range of temperatures between $313 \mathrm{~K}$ and $653 \mathrm{~K}$. At the same time, we are not aware of any available experimental data on the heat capacity of $\mathrm{Zr}_{3} \mathrm{Fe}$ intermetallic phase. Moreover, it is worth noting that there is no available information on standard entropies for these intermetallic phases.

Thermodynamic modeling of $\mathrm{Zr}-\mathrm{Fe}$ system without information on the thermodynamic properties of the phases becomes unreliable. At least, thermodynamics of the intermetallic compounds, that are stable in the wide temperature rage, like $\mathrm{Zr}_{3} \mathrm{Fe}$ and $\mathrm{ZrFe}_{2}$, should be defined for further improvement of the thermodynamic description of the $\mathrm{Zr}-\mathrm{Fe}$ system. In this respect, the topic of the next generation of databases of materials properties created based on CALPHAD approach in combination with reasonable description of thermodynamic properties starting from zero temperature attracts increasing attention. However, the experimental measurements of thermodynamic properties of materials at such low temperatures are rather difficult and timeconsuming; and most of all they are very expensive. On the other hand, the predictive power of state-of-the-art first-principles simulations has greatly improved, and they can be used efficiently to describe the thermodynamic properties of materials [18]. For example, the DFT approach in combination with quasi-harmonic approximation allows one to simulate the materials thermodynamic and thermal properties, such as heat capacity, entropy, etc. However, the QHA has its limitation, since it does not account for the anharmonic effects, which might be significant at high temperature [19]. Thus, to define the heat capacities of studied $\mathrm{Zr}_{3} \mathrm{Fe}$ and $\mathrm{C} 15-\mathrm{ZrFe}_{2}$ compounds we apply DFT+QHA method for the low-temperature regions and experimental measurements for the high temperatures. The reliability of DFT+QHA results and the boarders between low- and high-temperature regions are defined from the comparison between theoretical 
and experimental data. In addition to the heat capacity, we have determined the formation enthalpies and standard entropies of both compounds.

\section{Methods}

\subsection{Calculation of thermodynamic properties}

The total free energy $G_{\text {tot }}(V, T)$ of a system as a sum of electronic, vibrational and magnetic terms:

$$
G_{\text {tot }}(V, T)=E_{\text {el }}(V)-T S_{\text {el }}(V, T)+G_{\text {vib }}(V, T)+G_{m a g n}(V, T)
$$

considering each term as a function of volume $V$ and temperature $T$. In Eq. 1, the terms $\left[E_{\mathrm{el}}(V)-\right.$ $\left.T S_{\text {el }}\right], G_{\text {vib }}(V, T)$ and $G_{\text {magn }}(V, T)$ denote electronic, vibrational, and magnetic contributions to the total free energy, respectively. $\mathrm{Zr}_{3} \mathrm{Fe}$ phase is non-magnetic; therefore, the last term in the righthand side of Eq. 1 can be omitted in this case.

The electronic entropy can be estimated as:

$$
S_{\mathrm{el}}=-k_{B} \int_{-\infty}^{\infty} n(\varepsilon)[f \ln f+(1-f) \ln (1-f)] d \varepsilon
$$

Here, $n(\varepsilon)$ is the electronic density of states, and $f=f\left(\varepsilon, \varepsilon_{F}, T\right)$ is the Fermi distribution function. Vibrational (or phonon) contribution $G_{\text {vib }}(V, T)$ to the free energy was simulated within quasiharmonic approximation (QHA) where the phonon frequencies $\omega_{q}$ were derived from interatomic Hellmann-Feynman forces induced by finite atomic displacements. In QHA, the phonon frequencies are volume-dependent parameters, hence vibrational energy $G_{\text {vib }}$ becomes volumedependent, which makes it possible to account for the thermal expansion effects in the lattice. The vibrational energy as function of volume and temperature can be defined as:

$$
G_{\text {vib }}(V, T)=\sum_{q}\left\{\frac{\hbar \omega_{q}(V)}{2}+k_{B} T \ln \left[1-\exp \left(-\frac{\hbar \omega_{q}(V)}{k_{B} T}\right)\right]\right\}
$$

where $\omega_{q}$ is the phonon frequency with wave vector $q$, and $k_{\mathrm{B}}$ is the Boltzmann constant.

At low temperatures, which are of primary interest for theoretical simulations in this study, the dominant magnetic excitations are spin waves. A contribution from the spin-waves excitations to the total free energy of $\mathrm{ZrFe}_{2}$ phase was taken into consideration within Heisenberg model with nearest neighbors interactions. According to the model, the magnon-dispersion relations can be calculated as:

$$
\hbar \omega_{k}=4 J S[1-\cos (k a)](4)
$$

where $J$ denotes an effective (pair) exchange interaction between the nearest neighbors; $S$ is the on-site spin value of atom; $\omega_{k}$ is a magnon frequency with wave vector $k$; and $a$ is a lattice parameter. Computation of the $J$ parameter has been performed using the Exact Muffin-Tin Orbitals (EMTO) method [20], where the exchange parameter $J$ can be found analytically from the change in energy of embedding spins induced by rotation of them an infinitely small angle [21-23]. 
At the low temperatures, i.e. in the long wavelength limit $k \rightarrow 0$, the magnon dispersion has a parabolic form: $\hbar \omega_{k}=D_{\text {stiff }} \cdot k^{2}$, where $D_{\text {stiff }}=2 S J a^{2}$ denotes the spin-wave stiffness at $\mathrm{T}=0 \mathrm{~K}$. According to Kittel [24], at the low temperatures the internal energy of magnons $E_{\text {mag }}$ can be expressed as:

$$
E_{\text {mag }}(V, T)=\frac{k_{B} T}{2 \pi^{2}}\left(\frac{k_{B} T}{D_{\text {stiff }}}\right)^{\frac{3}{2}} \int_{0}^{x_{\max }} \frac{x^{\frac{3}{2}}}{\left(e^{x}-1\right)^{d x}}
$$

where $x=\frac{\hbar \omega_{k}}{k_{B} T}$. Since the effective exchange interaction $J$ is volume-dependent parameter, the spin-wave frequency also becomes volume-dependent $\omega_{k}=\omega_{k}(V)$, which allows one to implicitly calculate the magnon energy $E_{\operatorname{mag}}$ as a function of volume $V$.

The evaluation of the spin-wave free energy $G_{\mathrm{mag}}(V, T)$ can be done numerically by means of the internal energy $E_{\mathrm{mag}}(V, T)$ via:

$$
G_{\text {mag }}(V, T)=E_{\text {mag }}(V, 0)-T \int_{0}^{T} \frac{1}{T}\left(\frac{\partial E_{\text {mag }}(V, T)}{\partial T}\right)_{P} d T
$$

By knowing the total free energy $G_{\text {tot }}$ (see Eq. 1) as a function of volume $V$ and temperature $T$ it is possible to find the minimum free energy and corresponding volume $V_{\min }$ at each temperature. Thus, having the information on the ground state volume $V_{\min }$ as a function of the temperature we can determine the volumetric thermal expansion coefficient as:

$$
\alpha_{V}=\frac{1}{V_{0}} \frac{d V_{\min }}{d T}
$$

where $V_{0}$ denotes the ground state volume at $T=0 \mathrm{~K}$.

Bulk modulus as a function of the temperature can be expressed as:

$$
B(T)=V_{\text {min }}(T)\left(\frac{\partial^{2} G}{\partial V^{2}}\right)_{T}
$$

Specific heat capacity at constant volume, i.e. isochoric heat capacity, can be defined by formula given below:

$$
C_{V}=-T\left(\frac{\partial^{2} G}{\partial T^{2}}\right)_{V 0}
$$

Further, using the parameters obtained from Eqs. (7) - (10) one can determine the heat capacity at constant pressure, i.e. isobaric specific heat capacity, as:

$$
C_{P}=C_{V}+\alpha_{V}^{2} B_{T} V_{m} T
$$

where $V_{\mathrm{m}}$ denotes the molar volume of the unit cell.

An electronic contribution to the heat capacity can be expressed from electronic entropy as: 


$$
C_{e l}=T\left(\frac{\partial S_{e l}}{\partial T}\right)_{P}
$$

The spin-waves contribution to the heat capacity can be defined as the temperature derivative of $E_{\text {mag }}(V, T)$ :

$$
C_{m a g}=\left(\frac{\partial E_{m a g}}{\partial T}\right)_{P}
$$

\subsection{Calculations details}

Based on the previous experimental data [2], the $\mathrm{Zr}_{3} \mathrm{Fe}$ and $\mathrm{ZrFe}_{2}$ phases were simulated as orthorhombic and cubic C15 crystals, respectively, with 16 and 6 atoms per unit cell. In Fig.1, we depict the unit cells of these intermetallic phases. Thermodynamic properties of $\mathrm{Zr}_{3} \mathrm{Fe}$ and $\mathrm{ZrFe}_{2}$ phases were calculated using projector augmented wave (PAW) [25] potentials implemented in Vienna Ab-initio Simulation Package (VASP) [26]. The exchange-correlation effects were treated using the generalized gradient approximation [27]. Magnetic properties were accounted for within the collinear picture. The magnitude and orientations of the collinear local moments were calculated self-consistently. The cutoff energies for plane waves were set to 700 and $500 \mathrm{eV}$ for $\mathrm{Zr}_{3} \mathrm{Fe}$ and $\mathrm{ZrFe}_{2}$ compounds, correspondingly. The integration over the irreducible part of Brillouin zone has been carried out using Monkhorst-Pack method [28] on the grids of $18 \times 6 \times 6 \mathrm{k}$-points for $\mathrm{Zr}_{3} \mathrm{Fe}$ and $8 \times 8 \times 8$ for $\mathrm{ZrFe}_{2}$. The convergence criterion for electronic subsystem was chosen to be equal to $10^{-3} \mathrm{eV} /$ atom for subsequent iterations. The relaxation of atomic positions was realized by calculation of Hellman-Feynman forces [29,30] and stress tensor with using the conjugated gradient method. Relaxation was stopped when the forces became on the order of $10^{-3} \mathrm{eV} / \AA \dot{\text {. }}$.

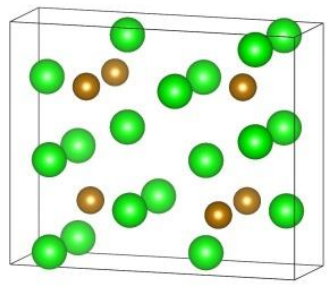

(a)

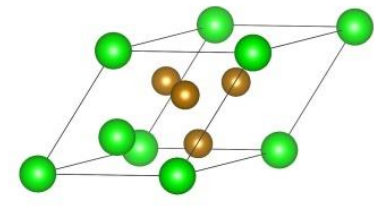

(b)

Figure 1 - Unit cells of orthorhombic $\mathrm{Zr}_{3} \mathrm{Fe}$ (a) and cubic $\mathrm{C} 15-\mathrm{ZrFe}_{2}$ (b) intermetallic compounds.

Regarding to the phonon calculations, we applied supercells approach where $\mathrm{Zr}_{3} \mathrm{Fe}$ and $\mathrm{ZrFe}_{2}$ crystals were simulated as $3 \times 2 \times 2$ (192 atoms) and $2 \times 2 \times 2$ (48 atoms) supercells, respectively. To calculate the interatomic forces in supercells we set the values of atomic displacements to be equal to 0.01 Á. Postprocessing calculations of phonons was realized within Phonopy code [31]. To check the accuracy of phonon calculations we considered the larger in size supercells, $4 \times 2 \times 2$ for $\mathrm{Zr}_{3} \mathrm{Fe}$ and $3 \times 3 \times 3$ for $\mathrm{ZrFe}_{2}$, and performed convergence test of heat capacities at the ground state volume. The test revealed that difference in heat capacities $C_{V}$ between larger and smaller supercells is about $\sim 1.5 \%$ at low-temperatures between 0 and $5 \mathrm{~K}$; and this difference gradually 
vanishes for the higher temperatures. Therefore, further phonon calculations were realized only for smaller in size supercells, e.i. $3 \times 2 \times 2$ for $\mathrm{Zr}_{3} \mathrm{Fe}$ and $2 \times 2 \times 2$ for $\mathrm{ZrFe}_{2}$ phase. Total free energy $G_{\text {tot }}$ from Eq. (1) was calculated for the 7 volume points in the range between -6 and $+6 \%$ with respect to the ground state volume at zero temperature.

Computation of effective exchange parameter $J$ in cubic $\mathrm{C} 15$ phase was performed using the EMTO method [20]. Simulations were carried out for a basis set including the valence $s, p, d$ and $f$ orbitals and by means of the frozen-core approximation, i.e. the core states were kept fixed. The integration over the irreducible part of the Brillouin zone has been performed using a grid of $6 \times 6 \times 6 \mathrm{k}$-points. The energy integration has been carried out in the complex plane using a semielliptic contour comprising 12 energy points. The convergence of the energy with respect to the calculation parameters has been set to $10^{-4} \mathrm{eV}$.

\subsection{Experimental methods}

\subsubsection{Sample preparation}

Two binary Fe-Zr alloys with the nominal compositions of $\mathrm{ZrFe}_{2}$ and $\mathrm{Zr}_{3} \mathrm{Fe}$ were prepared by arc-melting method in the Ar-atmosphere. Pieces of Fe (99.99\%, Alfa Aesar) and slugs of $\mathrm{Zr}$ (99.5\%, Alfa Aesar) were weighed in accordance to the nominal compositions. In order to achieve a good homogeneity of the chemical composition in the bulks, the samples were turnedover and re-melted three times. The samples were sealed in the quartz tubes with the reduced $\mathrm{Ar}$ atmosphere. The pressure of the Ar in the quartz tubes was chosen in order to reach $1 \mathrm{~atm}$. at the homogenization temperature. Afterwards, the samples were homogenized during 3 hours at 873 $\mathrm{K}$ for $\mathrm{Zr}_{3} \mathrm{Fe}$ and $1123 \mathrm{~K}$ for $\mathrm{ZrFe}_{2}$.

\subsubsection{Sample treatment and characterization}

The samples were analysed by X-ray diffraction (XRD) and scanning electron microscopy combined with an energy dispersive X-ray spectrometry (SEM/EDX).

Phase assemblages of specimens after homogenization annealing have been identified by X-ray powder diffraction (XRD) using the URD63 diffractometer (Seifert, FPM, Freiberg, Germany) equipped with the graphite monochromator and the $\mathrm{CuK}_{\alpha}$ radiation $(\lambda=1.5418 \AA$ ). The goniometer of the diffractometer had the Bragg-Brentano geometry. The Rietveld refinement was applied for the characterisation of all measured diffraction patterns using Maud software [28].

We have investigated the sample microstructures after homogenization annealing using scanning electron microscopy combined with dispersive X-ray spectrometry (SEM/EDX; Leo1530, Carl Zeiss/ Bruker AXS Mikroanalysis $\mathrm{GmbH}$ ). Chemical compositions of samples and present phases have been determined using a signal from EDX detector with an accuracy of \pm 2 at. \%.

Based on the XRD and SEM/EDX results the lattice parameters of $\mathrm{Zr}_{3} \mathrm{Fe}$ were found to be $a=$ $3.322 \AA, b=10.973 \AA, c=8.822 \AA$; and lattice parameter of $\mathrm{C} 15-\mathrm{ZrFe}_{2}-a=7.075 \AA$. XRD patterns are presented in the Fig.2. Measured lattice parameters exhibit a good agreement with the literature data [33-36]; and correspond to the stoichiometric intermetallic compounds. Microstructural analysis confirms the XRD results. Chemical composition of the samples measured by EDX method matches to the initial chemical composition within the error of the 
measurement. Chemical composition analysis shows that $\mathrm{Zr}_{3} \mathrm{Fe}$ samples have 77 at.\% of $\mathrm{Zr}$; and $\mathrm{ZrFe}_{2}$ samples -34 at.\% of $\mathrm{Zr}$. In both cases, we observed the deviations toward $\mathrm{Zr}$ side. However, the deviation was in the range of the reliable accuracy.

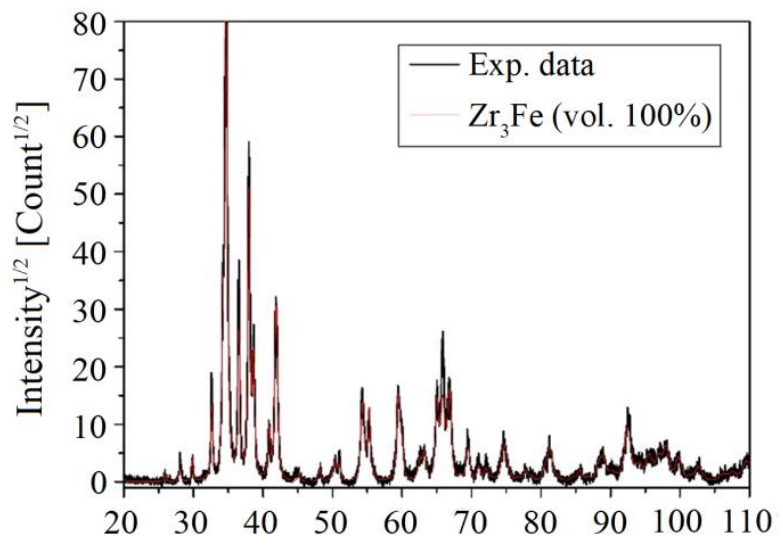

(a)

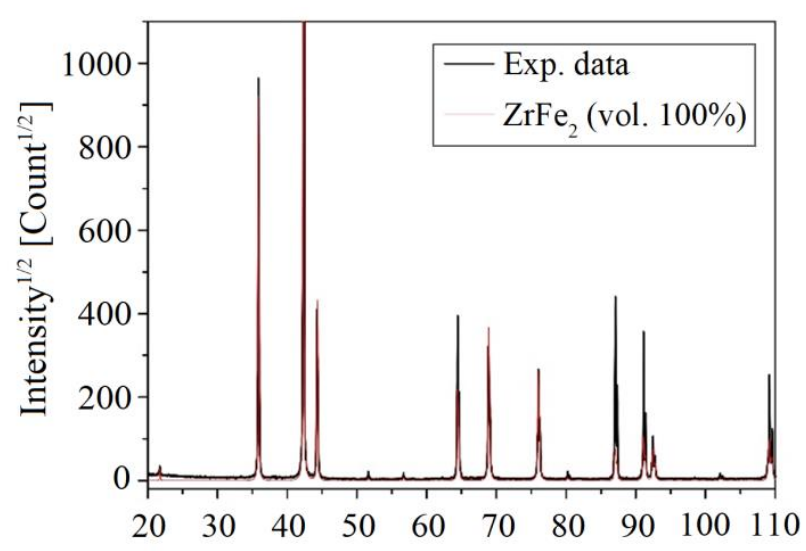

(b)

Figure 2 - XRD results of $\mathrm{Zr}_{3} \mathrm{Fe}$ (a) and $\mathrm{C} 15-\mathrm{ZrFe}_{2}$ (b) intermetallic compounds.

\subsubsection{Thermal expansion measurements}

In order to verify thermal expansion coefficients calculated in the frame of the QHA, additional experimental measurements of the thermal expansion of the investigated compounds have been performed. The thermal expansion measurements were carried out in the temperature ranges: 353-1023 $\mathrm{K}$ for $\mathrm{Zr}_{3} \mathrm{Fe}$ and 373-1523 $\mathrm{K}$ for $\mathrm{ZrFe}_{2}$. In total, two different dilatometric devices were involved, namely DIL 802 (Netzsch GmbH, thermocouple type S, Ar flow, $10 \mathrm{~K} / \mathrm{min}$ ) and DIL 805A/D/T (TA Instruments Inc. formerly Bähr-Thermoanalyse $\mathrm{GmbH}$, thermocouple type $\mathrm{K}$, vacuum atmosphere, heating rate $300 \mathrm{~K} / \mathrm{min}$ ). Temperature correction of DIL 802 was performed using temperatures of magnetic and solid transformations in Fe (99.95\% Alfa Aesar) and Co (99.995\% Alfa Aesar). Certified reference material of sapphire (Bähr-Thermoanalyse $\mathrm{GmbH}$ ) was used for calibration of linear temperature expansion. In the case of DIL 805A/D/T system, no additional calibration is required due to the specific design of the experimental procedure, since the thermocouple is placed directly onto the sample via spot welding. The measurements of two different samples were repeated two times with maximal uncertainty $6 \%$. In order to avoid possible temperature gradients in the sample, we used quenching mode of DIL805A/D/T with hollow quartz push rods. Due to the principle of induction heating, only the sample is heated during the measurement.

\subsubsection{Calorimetric measurements}

For heat capacity measurements we used the classical three-step method (continuous method) with a constant heating rate [37]. The measurements were performed in the wide range of temperatures using two different devices. In the temperature range from $235 \mathrm{~K}$ to $675 \mathrm{~K}$ we have used DSC 8000 device (Perkin Elmer, Pt/Rh crucible, He/Ar flow, heating rate $10 \mathrm{~K} / \mathrm{min}$ ). The measurements in the temperature range from $235 \mathrm{~K}$ to $675 \mathrm{~K}$ were divided into smaller intervals $100-150 \mathrm{~K}$. The heat capacity measurements in the temperature range from $623 \mathrm{~K}$ to $1220 \mathrm{~K}$ were performed in the one step using the device DSC Pegasus 404C (NETZSCH, Pt/Rh crucible, 
Ar flow, heating rate $10 \mathrm{~K} / \mathrm{min}$ ). Calibration was performed using the certified standard materials depending on the reliable temperature range of their heat capacity: copper standard was used in the temperature range from 100 to $320 \mathrm{~K}$; molybdenum - 300 to $673 \mathrm{~K}$ and platinum - 573 to $1473 \mathrm{~K}$. The measurements of two different samples were repeated two times with maximal uncertainty value of $3 \%$. It should be mentioned that at high temperatures the $C_{P}$ measurement with DSC equipment becomes less reliable due to increase of heat radiation which decreases a registered signal. This effect was considered during interpretation of the experimental data.

\section{Results}

\subsection{Zero temperature electronic structure calculations}

Let us start with calculations of thermodynamic properties of $\mathrm{Zr}_{3} \mathrm{Fe}$ and $\mathrm{ZrFe}_{2}$ phases at zero temperature. Theoretical data on lattice parameters, magnetic moments and formation energies for both phases are given in Table 1. Here, for comparison we also show the available experimental results at room temperature [34,35]. For orthorhombic $\mathrm{Zr}_{3} \mathrm{Fe}$ phase, the calculated lattice parameters $a$ and $b$ are slightly underestimated, while parameter $c$ is slightly overestimated compared to experimental data [34]. Simultaneously, the theoretical volume of $\mathrm{Zr}_{3} \mathrm{Fe}$ is in excellent agreement with experiment. $\mathrm{Zr}_{3} \mathrm{Fe}$ is found to be non-magnetic at zero temperature. For cubic $\mathrm{C} 15-\mathrm{ZrFe}_{2}$ phase, the calculated lattice parameter $a$ is $7.065 \AA$, while experiment gives $a=7.061 \AA$ [35]. This compound is found to be ferrimagnetic with average magnetic moment $1.12 \mu_{B}$ /atom, which is in good agreement with experimental result 1.04 $\mu_{B}$ /atom measured at the liquid helium temperature [38]. The calculated magnetic moments on Fe atoms with the average value of $1.924 \mu_{B}$ are parallel to each other, but antiparallel to magnetic moments induced on $\mathrm{Zr}$ atoms with the values of $-0.501 \mu_{B}$.

Formation enthalpies of the compounds are also listed in Tab. 1. They were calculated with respect to pure elements: non-magnetic hcp $\mathrm{Zr}$ and ferromagnetic bcc Fe. Our theoretical data on formation enthalpy $-0.121 \mathrm{eV} /$ at $(-11.67 \mathrm{~kJ} / \mathrm{mol})$ and $-0.288 \mathrm{eV} / \mathrm{at}(-27.79 \mathrm{~kJ} / \mathrm{mol})$ for $\mathrm{Zr} 3 \mathrm{Fe}$ and $\mathrm{ZrFe}_{2}$ phases, respectively, are in good agreement with previous DTF calculations $[15,16]$ and experimental results for $\mathrm{ZrFe}_{2}$ [11-14].

Table 1 - Ground state properties of $\mathrm{Zr}_{3} \mathrm{Fe}$ and $\mathrm{ZrFe}_{2}$ compounds at $\mathrm{T}=0 \mathrm{~K}$ and available experimental data.

\begin{tabular}{|c|c|c|c|c|}
\hline & \multicolumn{2}{|c|}{$\mathrm{Zr}_{3} \mathrm{Fe}$} & \multicolumn{2}{|c|}{$\mathrm{C}_{15-\mathrm{ZrFe}}$} \\
\hline & Theory & Exp. [34] & Theory & Exp. [35] \\
\hline $\begin{array}{c}\text { Lattice } \\
\text { parameter, } \AA\end{array}$ & $\begin{array}{c}\mathrm{a}=3.300 \\
\mathrm{~b}=10.900 \\
\mathrm{c}=8.945\end{array}$ & $\begin{array}{c}a=3.321 \\
b=10.966 \\
c=8.825\end{array}$ & $\mathrm{a}=7.065$ & $\mathrm{a}=7.061$ \\
\hline $\begin{array}{l}\text { Volume, } \\
\AA^{3} / \text { atom }\end{array}$ & 20.100 & 20.087 & 14.694 & 14.669 \\
\hline $\begin{array}{l}\text { Atomic basis } \\
\text { coordinates }\end{array}$ & 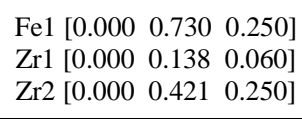 & 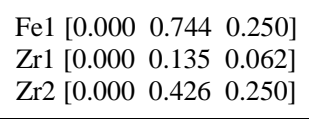 & $\begin{array}{l}\mathrm{Fe} 1\left[\begin{array}{lll}0.625 & 0.625 & 0.625\end{array}\right] \\
\mathrm{Zr} 1\left[\begin{array}{lll}0.000 & 0.000 & 0.000\end{array}\right]\end{array}$ & 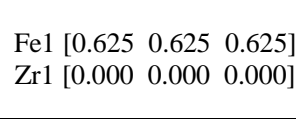 \\
\hline $\begin{array}{c}\text { Magnetic } \\
\text { moments, } \\
\mu_{\mathrm{B}} / \text { atom }\end{array}$ & Non-magnetic & Non-magnetic & $\begin{array}{lr}\text { Total: } & 1.12 \\
\text { Fe: } & 1.924 \\
\text { Zr: } & -0.501\end{array}$ & $\begin{array}{c}\text { Total: } 1.04 \text { [38] } \\
- \\
-\end{array}$ \\
\hline
\end{tabular}




\begin{tabular}{|c|c|c|c|c|}
\hline & & & $-0.308(-29.72)[11]$ \\
$\begin{array}{c}\text { Formation } \\
\text { energy, eV/at } \\
\text { (kJ/mole) }\end{array}$ & $-0.121(-11.62)$ & - & $-0.288(-27.79)$ & $-0.256(-24.70)[12]$ \\
& & & & $-0.228(-22.00)[13]$ \\
\hline
\end{tabular}

\subsection{Finite-temperature effects}

Because of the limitations of the experimental techniques to obtain data on the thermodynamic properties of the studied materials at low temperature, we calculate their thermodynamic properties in the temperature range from $0 \mathrm{~K}$ up to room temperature. We have accounted for the finite-temperature effects by determining the contributions of electronic entropy $S_{\mathrm{el}}$, magnetic excitations $G_{\text {mag }}$ and vibrational energy $G_{\text {vib }}$ to the total free energy $G_{\text {tot }}$ of the system. The electronic entropy $S_{\text {el }}$ was estimated using Fermi distribution which depends on Fermi energy and temperature (see Eq. 2).

\subsubsection{Magnetic excitations in $\mathrm{ZrFe}_{2}$ phase}

According to zero-temperature calculations, the $\mathrm{ZrFe}_{2}$ phase is ferrimagnetic with opposite orientation of local magnetic moments on $\mathrm{Zr}$ and $\mathrm{Fe}$ atoms; and there are two types of magnetic moments in this phase. However, since the pure $\mathrm{Zr}$ atom on its own is non-magnetic, we assume that in $\mathrm{ZrFe}_{2}$ phase the magnetic moment on $\mathrm{Zr}$ is induced by iron atoms in the local environment. Thus, for calculations of the magnon-dispersion relations we considered only the Fe-Fe pair exchange interactions.

The plot of magnon-dispersion relations as a function of wave vector $k$ is shown in Fig. 3. Here, the value of effective exchange parameter $J$ between Fe-Fe pair in the nearest neighborhood is $J_{F e-F e}^{N N}=3.747 \mathrm{meV}$. For the next nearest $\mathrm{Fe}-\mathrm{Fe}$ pairs the exchange interaction becomes comparable too small $J_{F e-F e}^{N N N}=-0.194 \mathrm{meV}$. The spin value for $\mathrm{Fe}$ atoms was chosen to be $\mathrm{S}=2$. Therefore, in the long wavelength limit $k \rightarrow 0$, the theoretical value of spin-waves stiffness for $\mathrm{ZrFe}_{2}$ phase was found to be $D_{\text {stiff }} \approx 750 \mathrm{meV} \cdot \AA^{2}$.

A simple estimation of the magnetic transition temperature $T_{\mathrm{C}}$ can be performed using pair exchange interactions within the mean-field approximation, $T_{C}^{M F A}=\frac{2}{3} \frac{1}{k_{B}} \cdot \sum_{j} J_{F e-F e} \cdot m_{F e} m_{F e}$, where summation runs over the first coordination shell and on-site magnetic moment is denoted as $m_{\mathrm{Fe}}$. The obtained value for the Curie temperature $T_{\mathrm{C}}$ is $772 \mathrm{~K}$. This result is overestimated as compared to the experimental value of $585 \mathrm{~K}$ [17], however, such a deviation is typical for the first-principles calculations of $T_{\mathrm{C}}$. 


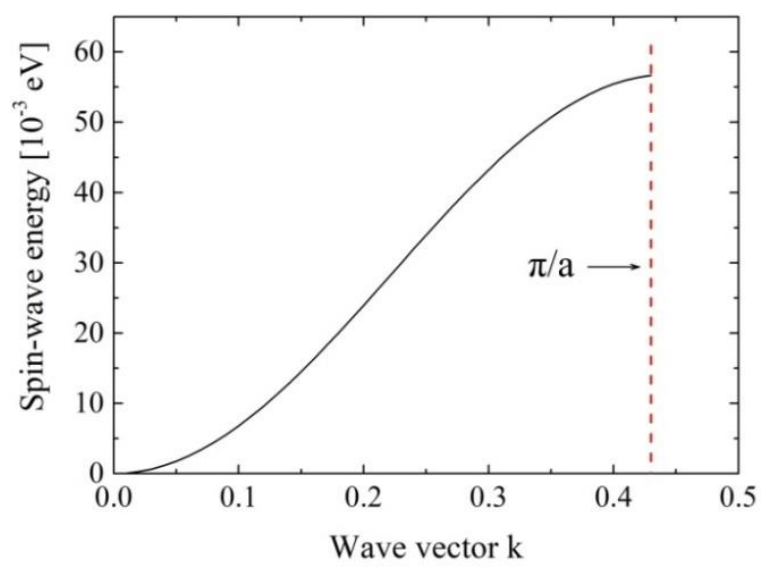

Figure 3 - Magnon-dispersion relations of $\mathrm{ZrFe}_{2}$ phase calculated for zero-temperature volume

\subsubsection{Phonon dispersion relations}

Calculated phonon dispersion relations and corresponding phonon density of states of $\mathrm{Zr}_{3} \mathrm{Fe}$ and $\mathrm{ZrFe}_{2}$ compounds are plotted in Fig. $4(\mathrm{a}, \mathrm{b})$. The dispersion curves of $\mathrm{Zr}_{3} \mathrm{Fe}$ and $\mathrm{ZrFe}_{2}$ are shown along $\Gamma-\mathrm{Z}-\mathrm{T}-\mathrm{Y}-\mathrm{S}-\mathrm{X}-\mathrm{U}-\mathrm{R}$ and $\Gamma-\mathrm{X}-\mathrm{W}-\mathrm{K}-\Gamma-\mathrm{L}$ directions of their Brillouin zones, respectively. The results confirm that both phases are dynamically stable since there are none imaginary frequencies for the whole set of wave vectors in the Brillion zones. According to the calculated phonon density of states (PDOS), the vibrational frequencies are distributed in the range between 0-7 THz for $\mathrm{Zr}_{3} \mathrm{Fe}$ and between $0-10 \mathrm{THz}$ for $\mathrm{ZrFe}_{2}$. This is related to the difference in the masses between $\mathrm{Zr}$ and $\mathrm{Fe}$ atoms. Thus, in the $\mathrm{Zr}_{2} \mathrm{Fe}$, which contains larger amount of $\mathrm{Fe}$ atoms with lighter atomic mass, the distribution of phonon frequencies is broader compared to $\mathrm{Zr}_{3} \mathrm{Fe}$ phase. By applying the Debye model to the calculated phonon frequencies we found the Debye frequency $\omega_{D}$ for each phase (see Fig. 4): $\omega_{D}\left(\mathrm{Zr}_{3} \mathrm{Fe}\right)=5.43 \mathrm{THz}$ and $\omega_{D}$ $\left(\mathrm{ZrFe}_{2}\right)=8.20 \mathrm{THz}$. Consequently, the Debye temperatures are $\theta_{D}\left(\mathrm{Zr}_{3} \mathrm{Fe}\right)=260.86 \mathrm{~K}$ and $\theta_{D}$ $\left(\mathrm{ZrFe}_{2}\right)=393.78 \mathrm{~K}$. The calculated values confirm our expectation on the reliability of the QHA for calculations of the thermodynamic properties of the studied systems in the temperature interval from $0 \mathrm{~K}$ to $300 \mathrm{~K}$. However, in some cases they will be shown up to higher temperatures for methodological reasons.

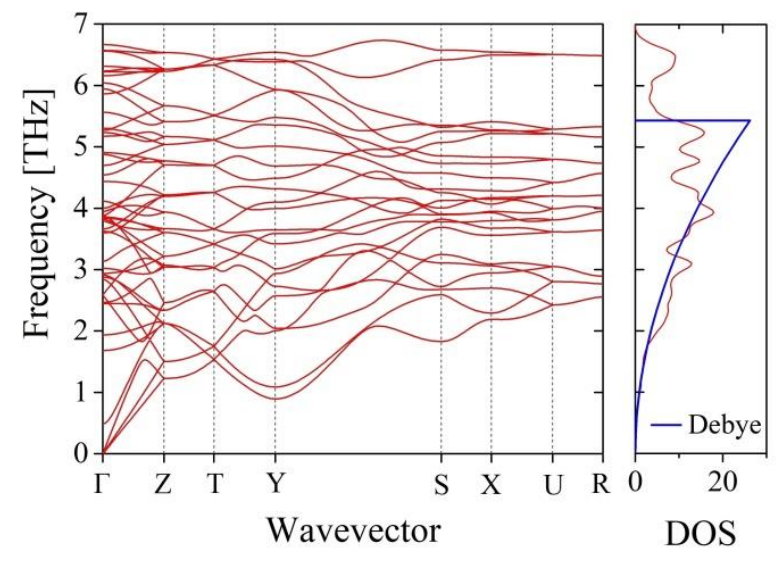

(a)

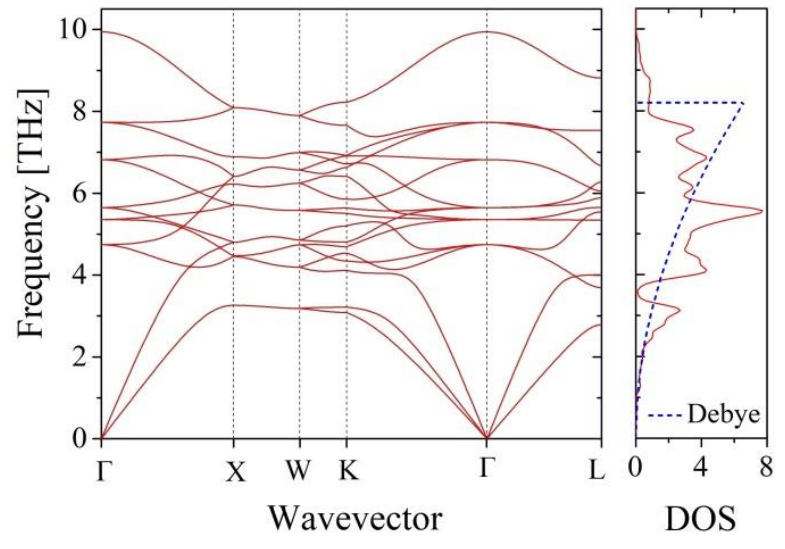

(b) 
Figure 4 - Phonon dispersion relations and phonon density of states of (a) $\mathrm{Zr}_{3} \mathrm{Fe}$ and (b) $\mathrm{ZrFe}_{2}$ compounds calculated for zero temperature volumes. Blue line represents the PDOS according to the Debye model.

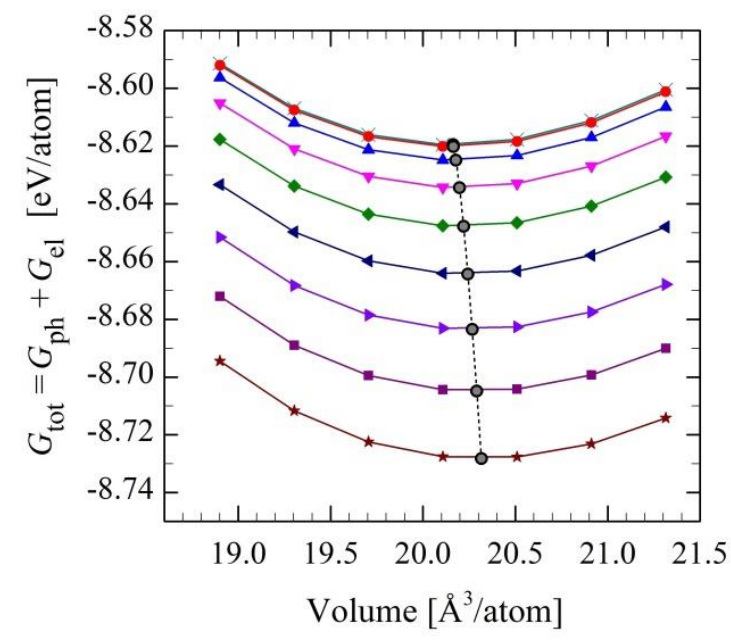

(a)

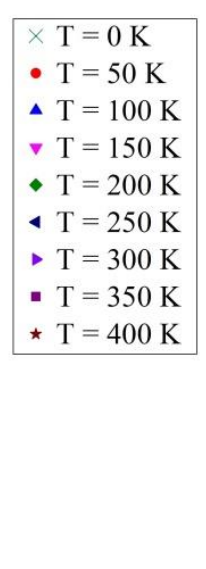

Figure 5 - Total free energy $G_{\text {tot }}$ of $\mathrm{Zr}_{3} \mathrm{Fe}$ (a) and $\mathrm{ZrFe}_{2}$ (b) phases plotted versus unit cell

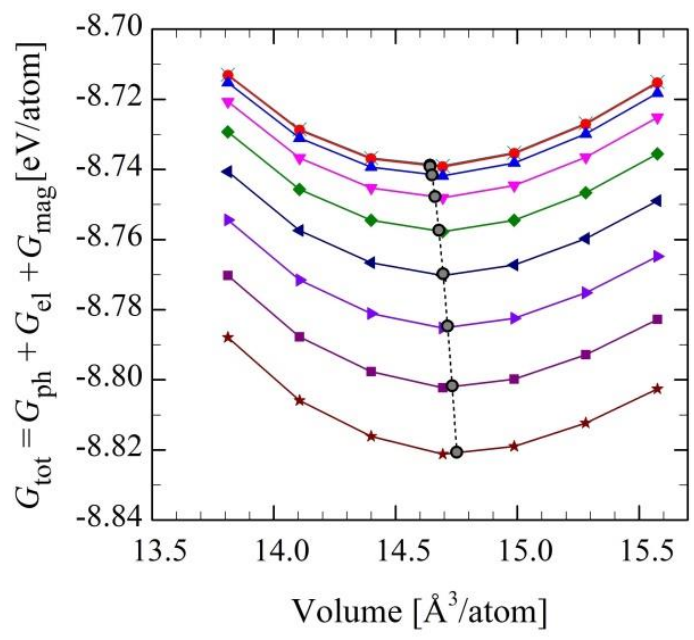

(b) volume for the range of temperatures. The dashed black lines represent equilibrium volume and energy (curves minima) for the range of temperatures.

Fig. 5 depicts calculated total free energies $G_{\text {tot }}$ of both phases as the function of volume $V$ and temperature $T$ in the range between $0 \mathrm{~K}$ and $400 \mathrm{~K}$. As can be seen from Fig. 5, the unit cell volumes, associated with the free energy minima, increase with the temperature. Dashed line, which connects the energy minima, shows the changes in ground state volume, viz. the thermal expansion of the lattices. Note that even at $\mathrm{T}=0 \mathrm{~K}$ we observe the slight deviation of ground state volume compared to zero temperature DFT calculations due to the zero-point vibrations (see Tab. 1). The corresponding volumes are $20.163 \AA^{3} /$ atom and $14.641 \AA^{3} /$ atom for $\mathrm{Zr}_{3} \mathrm{Fe}$ and $\mathrm{ZrFe}_{2}$, respectively. Interestingly, that the sign of this deviation is different for $\mathrm{Zr}_{3} \mathrm{Fe}$ and $\mathrm{ZrFe}_{2}$ phases.

\subsection{Thermal expansion coefficient}

Let us now consider the properties that can be determined from the total free energy and compare them to our experiment. The experimental dilatometry results on $\mathrm{Zr}_{3} \mathrm{~F}$ and $\mathrm{ZrFe}_{2}$ compounds obtained in this work and theoretical results on the volumetric thermal expansion coefficients calculated from Eq. 7 are summarized in Fig. 6. To find the volumetric thermal expansion coefficients from dilatometry results, it was assumed that both phases expand isotropically with temperature. Therefore, the volumetric coefficient is three times the linear coefficient: $\alpha_{V}=3 \alpha_{L}$.

According to the theoretical data, in the range of temperatures between 0 and $200 \mathrm{~K}$ the thermal expansion coefficient of $\mathrm{Zr}_{3} \mathrm{Fe}$ is slightly higher than the one for $\mathrm{ZrFe}_{2}$ phase. With further increasing of the temperature $\alpha_{V}$ of $\mathrm{ZrFe}_{2}$ phase would become higher within QHA.

However, a comparison of calculated and experimental results shows that QHA significantly underestimates the values of $\alpha_{V}$ at temperatures above $\theta_{D}$, where the contribution of anharmonic 
effects increases. Moreover, in the experiment we see that $\mathrm{ZrFe}_{2}$ compound exhibits the magnetic transition. The Curie temperature measured by thermal expansion measurements is in a good agreement with our DCS results (reported below) and with data from literature, $T_{\mathrm{c}}=585 \mathrm{~K}$ [17]. Because of this, for $\mathrm{ZrFe}_{2}$ the magnetic contribution to thermal expansion becomes totally dominant, leading to well-developed $\lambda$-peculiarity at the magnetic phase transition. As will be seen from DSC results, the beginning of our thermal expansion measurements for $\mathrm{ZrFe}_{2}$ is in the temperature range, where the magnetic transition already influences significantly on the thermodynamic properties of this compound. Therefore, the experimentally observed behavior of the thermal expansion coefficient of $\mathrm{ZrFe}_{2}$ compound in the temperature range of 353-450 K cannot be directly compared with theory, justifying our decision to limit the simulations to lowtemperatures. At the same time, one can see that the theoretical $\alpha_{V}$ of $\mathrm{Zr}_{3} \mathrm{Fe}$ agrees well with experiment (within measurement uncertainty $6 \%$ ) at the temperatures close to the room temperature, confirming the reliability of the adopted theoretical scheme below $T \sim 300 \mathrm{~K}$.

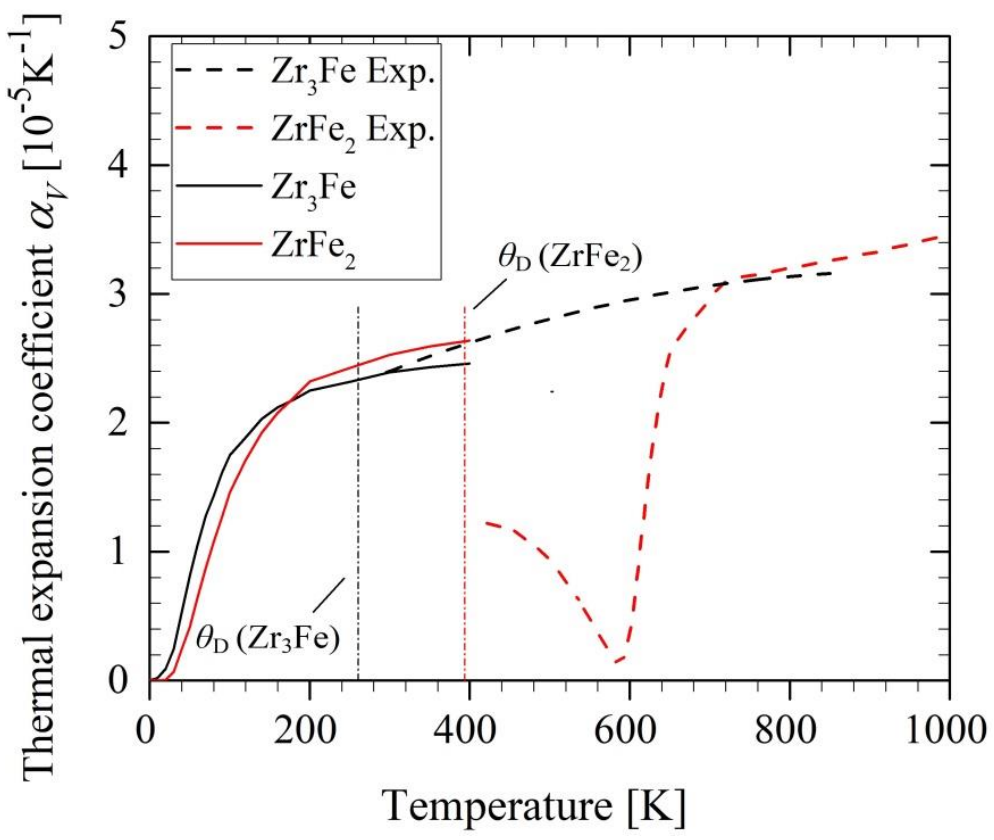

Figure 6 - Experimental (dashed lines) and calculated (solid lines) volumetric coefficient of thermal expansion of $\mathrm{Zr}_{3} \mathrm{Fe}$ (black) and $\mathrm{ZrFe}_{2}$ (red). Vertical lines denote the Debye temperatures $\theta_{D}$ of both compounds.

\subsection{Compressibility}

In Fig. 7 we show the calculated results for bulk modulus $B$ plotted as a function of temperature. In the range between $0 \mathrm{~K}$ and $300 \mathrm{~K}$, each phase exhibits the reduction of bulk modulus with temperature: by $\sim 3 \%$ for $\mathrm{Zr}_{3} \mathrm{Fe}$ and by $\sim 2 \%$ for $\mathrm{ZrFe}_{2}$. Unfortunately, there are no available experimental data on bulk modulus of $\mathrm{Zr}_{3} \mathrm{Fe}$ and $\mathrm{ZrFe}_{2}$ intermetallic compounds, however, our theoretical results are in good agreement with previous ab-initio calculations [15,39]. 


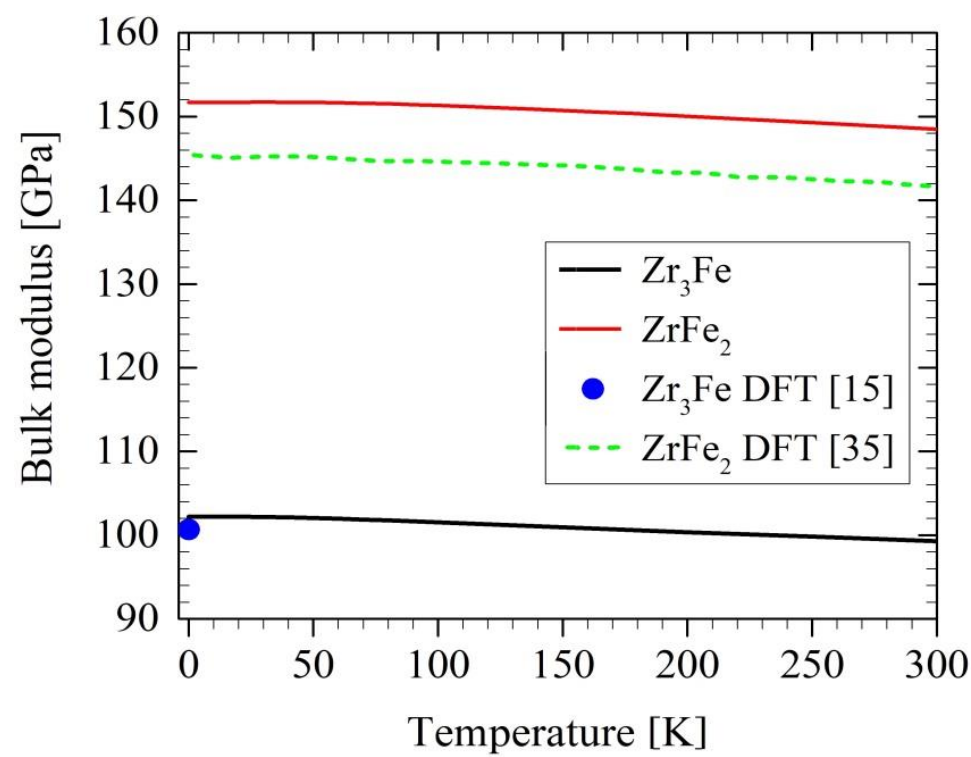

Figure 7 - Bulk modulus of $\mathrm{Zr}_{3} \mathrm{Fe}$ (black line) and $\mathrm{ZrFe}_{2}$ (red line) compounds plotted versus temperature.

\subsection{Heat capacity}

The measurements of heat capacities $C_{P}$ have been performed in range of temperatures between 100 and $1000 \mathrm{~K}$ for $\mathrm{Zr}_{3} \mathrm{Fe}$ phase and in the range between 100 and $1480 \mathrm{~K}$ for $\mathrm{ZrFe}_{2}$ phase. To the best of our knowledge, the measurements in such a broad temperature interval are reported for the first time. Results are plotted in Fig. 8. According to these data, the heat capacity $C_{P}$ of $\mathrm{ZrFe}_{2}$ phase exhibits a peak related to the magnetic transition at $585 \mathrm{~K}$. Our $C_{P}$ measurements of $\mathrm{ZrFe}_{2}$ phase accurately reproduce previous experimental results in the range between 315 and $655 \mathrm{~K}$ from Ref. [17]. Experimental heat capacity of $\mathrm{Zr}_{3} \mathrm{Fe}$ phase is plotted in Fig. 8a. Note that for orthorhombic $\mathrm{Zr}_{3} \mathrm{Fe}$ phase the $C_{P}$ was not studied before.

Theoretical calculations of total $C_{P}$ are also shown in Fig. 8. In the case of orthorhombic $\mathrm{Zr}_{3} \mathrm{Fe}$ phase (see Fig. 8a), at the low-temperature regions up to $300 \mathrm{~K}$ the theoretical heat capacity $C_{P}$ agrees within $\pm 3 \%$ with experimental data. For high temperatures the difference between theory (not shown) and experiment increases due to the increasing contribution from anharmonic effects. Regarding the $\mathrm{ZrFe}_{2}$ phase (see Fig. 8b), one can see that the theory matches with experiment (within $\pm 3 \%$ measurement error) in the range between 100 and $200 \mathrm{~K}$. At higher temperatures the difference between theory and experiment significantly increases, most probably due to magnetic transition at $T_{\mathrm{C}}=585 \mathrm{~K}$. In fact, the contribution of magnetic excitations from Heisenberg model (reported below) weakly affects the calculated total heat capacity of $\mathrm{ZrFe}_{2}$ phase. Moreover, we did not model the paramagnetic state of this compound, which strongly affects theoretical calculations of thermodynamic properties above $T_{\mathrm{C}}$ [40-42] Nonetheless, in the low-temperature regions where the anharmonic and magnetic effects are small, we observe very good agreement between theory and experiment. 


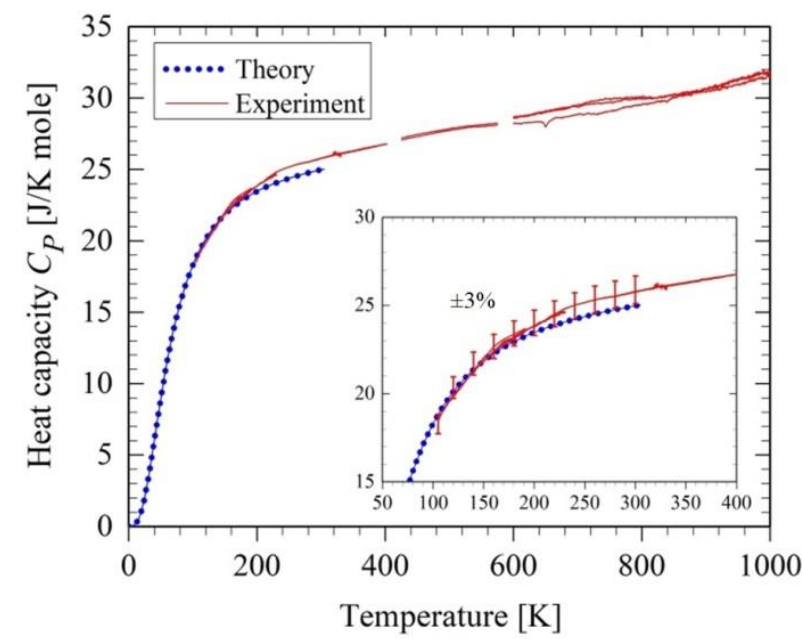

(a)

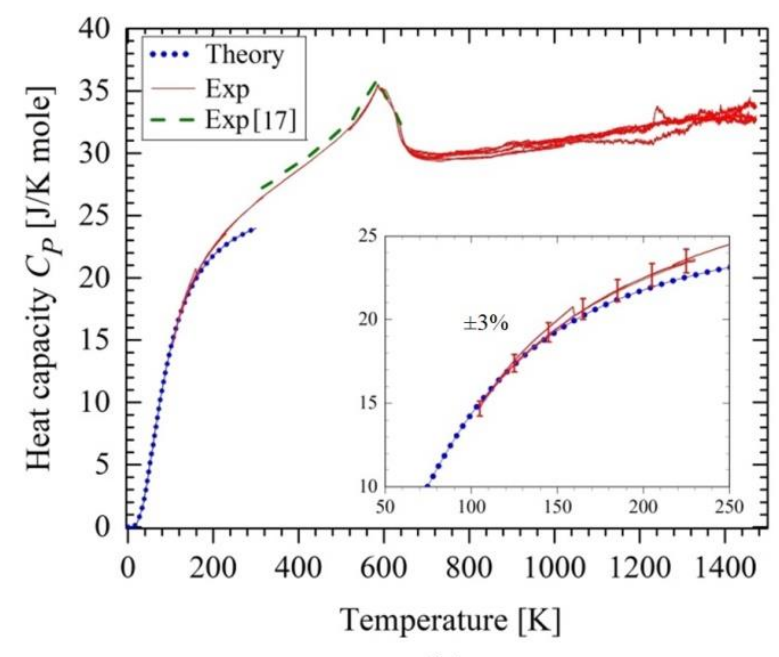

(b)

Figure 8 - Theoretical and experimental results on heat capacity of $\mathrm{Zr}_{3} \mathrm{Fe}$ (a) and $\mathrm{ZrFe}_{2}$ (b) intermetallic compounds. Black and red lines respectfully represent our theoretical and experimental data. Green dashed line represents the available experimental data from Ref. [17]. The insets show the low temperature regions with information on the experimental error.

In Fig. 9 we plot the calculated contributions of phonons, electrons and magnons to the heat capacity. Fig. 9a represents the vibrational heat capacities at constant volume $C_{V}^{p h}$ and pressure $C_{P}^{p h}$ as well as electronic heat capacity $C_{P}^{e l}$. One can see that with the temperature the isochoric heat capacity $C_{V}^{p h}$ (red dashed line) of $\mathrm{Zr}_{3} \mathrm{Fe}$ phase reaches the saturation values $\sim 25 \mathrm{~J} / \mathrm{K} \cdot \mathrm{mole}=$ $3 \mathrm{R}$ faster than the one for $\mathrm{ZrFe}_{2}$ phase. Isobaric heat capacity $C_{P}^{p h}$ was calculated using Eq. (10) and the information on $\alpha_{V}(T)$ and $B(T)$, discussed above. Regarding the electronic contribution, the $\mathrm{Zr}_{3} \mathrm{Fe}$ phase exhibits higher values of $C_{P}^{e l}$ compared to the $\mathrm{ZrFe}_{2}$ phase. In the lowtemperature limit up to $100 \mathrm{~K}$, calculated $C_{P}^{e l}$ of each phase changes linearly with temperature. This result in accordance with the Sommerfeld model for electron heat capacity described as $C_{V}^{e l}=\frac{\pi^{2}}{3} k_{B}^{2} N\left(\varepsilon_{F}\right) \cdot T=\gamma \cdot T$, where parameter $\gamma$ is equal to 0.0025 and 0.0011 for $\mathrm{Zr}_{3} \mathrm{Fe}$ and $\mathrm{ZrFe}_{2}$, respectively. Magnon heat capacity $C_{P}^{m a g} \sim T^{3 / 2}$ for $\mathrm{ZrFe}_{2}$ phase is plotted in Fig. 9b. Here, we compare $C_{P}^{\text {mag }}$ with $C_{P}^{e l}$ for $\mathrm{ZrFe}_{2}$; and as one can see, the spin-waves contribution to the total heat capacity is comparably too small. 


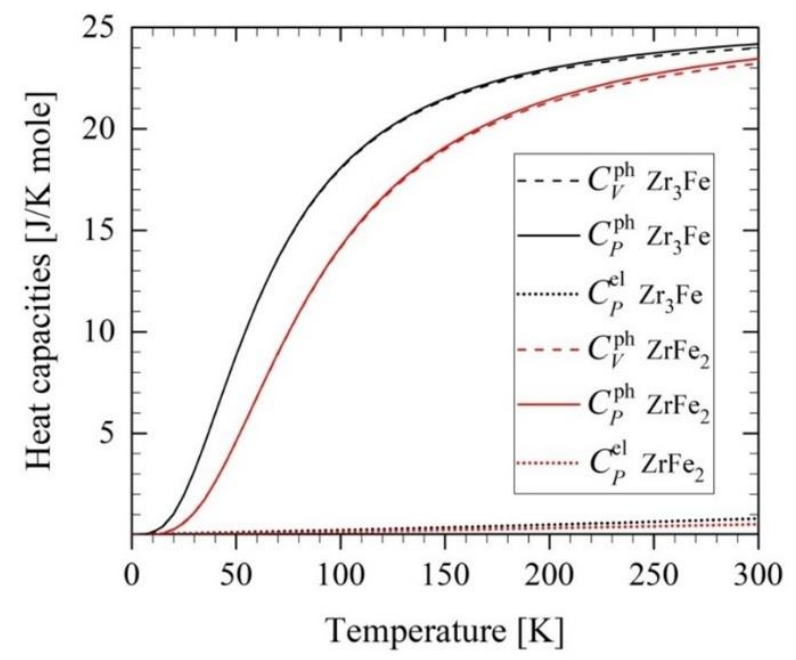

(a)

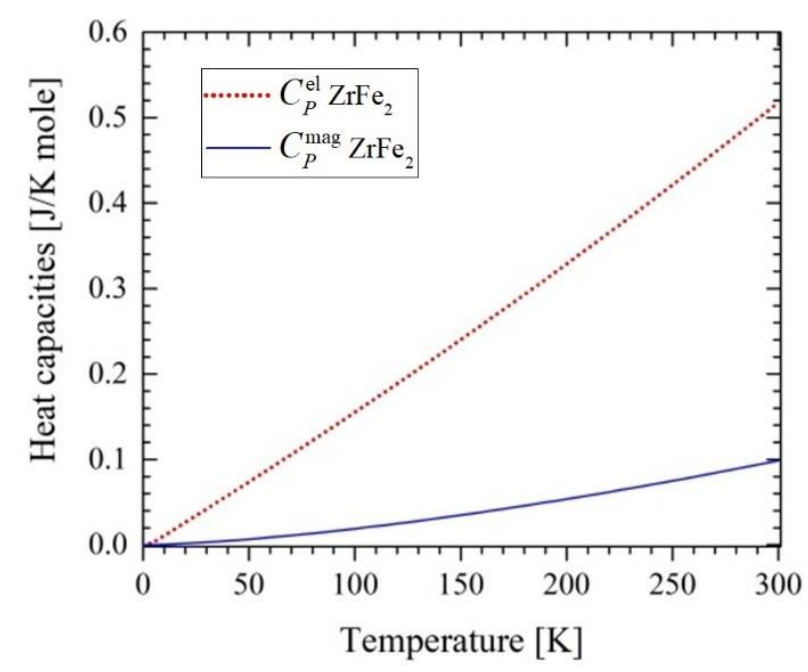

(b)

Figure 9 - (a) Calculated vibrational $\left(C_{V}^{p h}, C_{P}^{p h}\right)$ and electronic $C_{P}^{e l}$ contributions to heat capacity; as a function of temperature for $\mathrm{Zr}_{3} \mathrm{Fe}$ (black lines) and $\mathrm{ZrFe}_{2}$ (red lines) phases; and (b) comparison of spin-waves heat capacity $C_{P}^{m a g}$ with electronic heat capacity $C_{P}^{e l}$ in ferrimagnetic $\mathrm{ZrFe}_{2}$ phase.

\section{Discussion}

For $\mathrm{Zr}_{3} \mathrm{Fe}$ phase, where these is no magnetic transition, we observed a good agreement between theoretical and experimental heat capacities (see Fig. 8a). The most probable reason of deviation between theory and experiment might be the arising of anharmonic effects with the temperature. This assumption is supported by comparison of our theoretical and experimental data on thermal expansion coefficient (see Fig. 6a): QHA underestimates the thermal expansion of $\mathrm{Zr}_{3} \mathrm{Fe}$ phase at the temperatures above $\sim 300 \mathrm{~K}$. The same assumption could be applied for $\mathrm{ZrFe}_{2}$ phase according to data on thermal expansion and heat capacity (see Figs. 6b and 8b); however, the presence of magnetic transition makes it difficult to estimate the anharmonic effects on heat capacity of $\mathrm{ZrFe}_{2}$; and it is obvious from Fig. $8 \mathrm{~b}$ that the deviation between theoretical and experimental heat capacities mostly arises from magnetic transition in $\mathrm{ZrFe}_{2}$. Additionally, there are other factors that can influence on the deviation between theoretical and experimental $C_{P}$ such as vacancies and other defects. However, their estimations are beyond the scope of the present work. From the comparison between DFT+QHA and DSC data on $C_{P}$ we conclude that QHA results are reliable up to $300 \mathrm{~K}$ for $\mathrm{Zr}_{3} \mathrm{Fe}$ and $200 \mathrm{~K}$ for $\mathrm{ZrFe}_{2}$.

So, in the low-temperature region the QHA gives fairly accurate information on thermodynamic properties of $\mathrm{Zr}_{3} \mathrm{Fe}$ and $\mathrm{ZrFe}_{2}$ intermetallic compounds. This allows us to extrapolate our experimentally measured heat capacity to zero temperature using our theoretical data. That is to say, through the combination of theory and experiment we can accurately determine the heat capacity in the range from $0 \mathrm{~K}$ up to the highest studied temperature. To derive the equations for $C_{P}$ we used RW-model [43], which is traditional for thermodynamic databases:

$$
C_{P}^{R W}\left(T, \theta^{R W}\right)=C_{V}^{D e b}(T)+a T+b T^{2}+C_{P}^{m a g}(T)
$$


where $T$ is temperature, $\theta^{R W}=\left(\theta_{D}, a, b\right)$ is the vector of the unknown model parameters to be estimated, $C_{P}^{D e b}(T)$ is the heat capacity describing phonon contribution using Debye model, $C_{P}^{m a g}(T)$ is magnetic contribution of the heat capacity.

Magnetic contribution to the thermodynamic properties was taken into account according to Hillert and Jarl [44] in the following form:

$$
C_{P}^{m a g}=R \ln \left(\beta_{0}+1\right) g(\tau)
$$

where $\tau$ is $T / T^{*}, T^{*}$ is the critical temperature (the Curie temperature $\mathrm{T}_{\mathrm{C}}$ for ferromagnetic materials or the Neel temperature $T_{\mathrm{N}}$ for antiferromagnetic materials), $\beta_{0}$ the average magnetic moment per atom and $g(\tau)$ is a function depending on $\tau$.

Obtained thermodynamic RW-models well reproduce the experimental data at high temperatures, as well as theoretical results at low temperatures. Thus, based on the obtained fitted models of $C_{P}$, it is possible to determine the standard entropies of both phases using the expression $S^{298}=\int_{0}^{298} C_{P} \frac{d T}{T}$. The data on $C_{P}, S^{298}$ and $\Delta H_{f}$ for $\mathrm{Zr}_{3} \mathrm{Fe}$ and $\mathrm{ZrFe}_{2}$ phases are listed in Table 2.

Table 2 - Values of $C_{P}$ fitting parameter, $S^{298}$ and $\Delta H_{f}$ for $\mathrm{Zr}_{3} \mathrm{Fe}$ and $\mathrm{ZrFe}_{2}$ phases.

\begin{tabular}{|c|c|c|}
\hline Parameters $\backslash$ Compound & $\mathrm{Zr}_{3} \mathrm{Fe}$ & $\mathrm{ZrFe}_{2}$ \\
\hline$\theta_{D}, K$ & 260.86 & 393.78 \\
\hline$a \cdot 10^{3}$ & 5.62 & 6.85 \\
\hline$b \cdot 10^{7}$ & 10.52 & -8.79 \\
\hline$\beta_{0}$ & - & 0.466 \\
\hline$T_{C}, K$ & - & 585 \\
\hline$S^{298}, \mathrm{~J} / \mathrm{mole} \cdot \mathrm{K}$ & 38.4 & 30.8 \\
\hline$\Delta H_{f}, \mathrm{~kJ} / \mathrm{mole}$ & -11.62 & -27.79 \\
\hline
\end{tabular}

\section{Conclusions}

In this work, using the combination of DFT+QHA approach and experimental DSC analysis we obtained the main thermodynamic parameters $S^{298}$ and $\Delta H^{0}$ and functions $C_{P}(T)$ for $\mathrm{Zr}_{3} \mathrm{Fe}$ and $\mathrm{ZrFe}_{2}$ intermetallic phases form $0 \mathrm{~K}$ up to temperatures of their stability. Experimental measurements of heat capacity of both phases have been performed for the first time in wide range of temperatures. Analysis of theoretical and experimental data on $\alpha_{V}$ and $C_{P}$ shows that QHA remarkably underestimates the anharmonic and magnetic effects starting from temperatures 200-300 K. However, for low-temperature regions we observed very good agreement between theory and experiment. The new and complete information on the heat 
capacity and standard entropy and of $\mathrm{Zr}_{3} \mathrm{Fe}$ and $\mathrm{ZrFe}_{2}$ compounds is very important for future development of reliable thermodynamic description of the $\mathrm{Zr}-\mathrm{Fe}$ binary system. The present study allows to perform the thermodynamic modeling in terms of the $3^{\text {rd }}$ generation CALPHAD approach including the reasonable description of thermodynamic properties starting from zero temperature.

\section{Acknowledgments}

Theoretical calculations were supported by the Ministry of Science and High Education of the Russian Federation in the framework of Increase Competitiveness Program of NUST "MISIS" (No. K2-2019-001) implemented by a governmental decree dated 16 March 2013, No 211. We acknowledge support from the Strategic Research Areas the Swedish e-Science Research Centre (SeRC) and Advanced Functional Materials at Linköping University (Faculty Grant SFOMatLiU No. 2009 00971). The authors thank the German Research Foundation (DFG) for funding within the Collaborative Research Center SFB 799 Trip-Matrix Composites. A. Udovsky gratefully acknowledges Russian Foundation for Basic Research (No. 19-03-00530_a) for support in experimental measurements. In addition, we thank G. Savinykh. and Dr. C. Schimpf (TUFreiberg) for technical contribution.

\section{References}

[1] M.S. Granovski, D. Arias, J. Nucl. Mater. 229 (1996) 29-35.

[2] F. Stein, G. Sauthoff, and M. Palm, J. Phase Equilibria 23 (2002). https://doi.org/10.1361/105497102770331172

[3] X. Tao, J. Zhu, H. Guo, Yi. Ouyang, Yo. Du, J. Nucl. Mater. 440 (2013) 6-10.

[4] H. Biermann et al., Adv. Eng. Mater. 11 (2009) 1000-1006. https://doi.org/10.1002/adem.200900210

[5] S. Martin et al., Steel Research int. 82 (2011) 1133-1140.

[6] L.N. Guseva, T.O. Malakhova, X-Ray Investigations of Alloys of Iron with Zirconium, IronRich (Fe-ZrFe2). Metallofiz. (Kiev) 46 (1973) 111-113.

[7] T.O. Malakhova and A.N. Kobylkin, The Zr-Fe Phase Diagram (0-66.6 at.\% Fe). Russ. Metall. (1982) 187-191.

[8] K. Bhanumurthy and G.B. Kale, Scr. Metall. Mater. 28 (1993) 753-756.

[9] C. Guo, Z. Du, C. Li, B. Zhang, M. Tao, Calphad 32 (2008) 637-643.

[10] Yi. Yang, L. Tan, H. Bei, J.T. Busby, J. Nucl. Mater. 441 (2013) 190-202.

[11] J.C. Gachon, J. Hertz, Calphad 7 (1983) 1-12.

[12] A. Schneider, et al., Pure Appl. Chem. 2 (1961) 13-16.

[13] O.Yu. Sidorov, et al., Rasplavy 3 (1989) 28-33.

[14] M. Rosner-Kuhn, et al., Z. Metallkd. 86 (1995) 682-685.

[15] K. Ali, A. Arya, P.S. Ghosh, G.K. Dey, Comput. Mater. Sci. 112 (2016) 52-66.

[16] M. Mihalkovic, M. Widom, Phys. Rev. B 70 (2004) 144107.

[17] R. Lück, H. Wang, J. Alloys Compd. 191 (1993) 11-12.

[18] P.E.A. Turchi, et al., Calphad 31 (2007). https://doi.org/10.1016/j.calphad.2006.02.009

[19] B. Grabowski, L. Ismer, T. Hickel, and J. Neugebauer, Phys. Rev. B 79 (2009) 134106. 
[20] L. Vitos, Computational Quantum Mechanics for Materials Engineers: The EMTO Method and Applications, Springer-Verlag, London, (2007). DOI: 10.1007/978-1-84628-951-4

[21] A. I. Liechtenstein et al, J. Phys. F: Met. Phys. 14 (1984) L125.

[22] A. I. Liechtenstein et al, Solid State Commun. 54 (1985) 327.

[23] A. I. Liechtenstein et al, J. Magn. Magn. Mater. 67 (1987) 65.

[24] Ch. Kittel, Quantum theory of solids, $2^{\text {nd }}$ Revised Edition (1987). ISBN: 978-0-471-62412-7

[25] P. E. Blöchl, Projector augmented-wave method, Phys. Rev. B 50 (1994) 17953.

[26] G. Kresse, J. Furthmüller, Phys. Rev. B 54 (1996) 11169.

[27] J. P. Perdew, K. Burke, and M. Ernzerhof, Phys. Rev. Lett. 77 (1996) 3865.

[28] H. J. Monkhorst and J. D. Pack, Phys. Rev. B 13 (1976) 5188.

[29] H. Hellmann, Einführung in die Quantumchemie. Deuticke, Leipzig. (1937). https://doi.org/10.1002/ange.19410541109

[30] R.P. Feynman, Phys. Rev. 56 (1939) 340.

[31] A. Togo and I. Tanaka, Scr. Mater. 108 (2015) 1-5.

[32] L. Lutteroti, MAUD, CPD Newsletter, IUCr 24 (2000).

[33] W. Brueckner, K. Kleinstueck, G.E.R. Schulze, Physica Status Solidi. 23 (1967) 475-480.

[34] P. Matkovic, T. Matkovic, I. Vickovic, Metalurgija, Croatia. 29 (1990) 3-6.

[35] E. Piegger, R.S.Craig, J. Chemical Phys. 39 (1963) 137-145.

[36] H. Boller, Monatshefte fuer Chemie 104 (1973) 545-549.

[37] G. Gatta, et al., Pure Appl. Chem. 78 (2006) 1455-1476.

[38] C.W. Kocher and P.J. Brown, J. Appl. Phys. 33 (1962) 1091.

[39] K. Ali, P.S. Ghosh, A. Arya, J. Alloys Compd. 723 (2017) 611-619.

[40] B. Alling, T. Marten, and I. A. Abrikosov, Nature Materials 9 (2010) 283.

[41] P. Steneteg, B. Alling, and I. A. Abrikosov, Phys. Rev. B 85 (2012) 144404.

[42] N. Shulumba, et al., Phys. Rev. B 89 (2014) 174108.

[43] M.W. Chase, et al., Group 1: Heat capacity models for crystalline phases from $0 \mathrm{~K}$ to 6000

K. Calphad: Computer Coupling of Phase Diagramsand Thermochemistry 19 (1995) 437-447.

[44] M. Hillert, M. Jarl, Calphad 2 (1978) 227-238. 